\title{
Considerations on studying the characteristics of the elastic axle steering system on railway vehicles
}

\author{
Ioan Sebeșan ${ }^{1, *}$, Sorin Arsene ${ }^{1}$, and Mircea Radu Sebeșan ${ }^{2}$ \\ ${ }^{1}$ Polytechnic University of Bucharest, Sector 6, Splaiul Independenței, No. 313, Romania \\ ${ }^{2}$ SC Metrorex SA, Sector 1, Dinicu Golescu, No. 38, Romania
}

\begin{abstract}
The new orientation in construction of bogies consists in realization possibility "negotiation the radii of curvature", the axes being placed in an approximately radial position. This reduces wear on the wheel and rail surfaces if the stability of the horizontal transverse movements of the vehicle is not affected. In railway motor vehicles, these requirements are achieved by means of rubber elastic elements which by their own damping capacity also contribute to the reduction of axle movement. The paper presents a series of considerations regarding the elastic constants of the springs from the axle steering system.
\end{abstract}

\section{Introduction}

Of particular importance for ensuring a stable circulation and low forces wheel-rail friction it has the axle steering system. This must transmit interaction forces with the tread without impeding the operation of the suspension, while ensuring and a correct position of the axle in the track and towards the vehicle.

At high speeds, it turned out be more advantageous elastic driving of the axles both in the transverse and longitudinal directions.

The new orientation in the construction of bogies consists of making it possible to negotiate of the radii of curvature, that is, the quasi-radial settlement in the curve of the axle of the bogie. This reduces the wear of the wheel and rail contact surfaces, but it is necessary not to cause instability of the as hunting movements.

The tendency of radial settlement of an axle can be done if the rolling profile it is wear and the driving system is sufficiently elastic. An excessive elasticity of the axle steering springs negatively influences the hunting movement of the axle in the sense at it reduces the critical velocity of the hunting movement.

The problems presented are part of the theoretical and experimental studies conducted the coordination of Prof. Eng. Ioan Sebeşan, within the Department of Railway Rolling Stock of the Polytechnic University of Bucharest.

\footnotetext{
* Corresponding author: ioan_sebesan@yahoo.com
} 


\section{Influence of the elasticity of the driving system on the geometric alignment of the axle in the curves}

In fig. 1 shows the case of a bogie in which the axle driving arm 1 is connected to the bogie frame 2 by means of a silent-block 3, with possibilities of varying the longitudinal and transverse elasticity by conical tightening of the parts 4 .

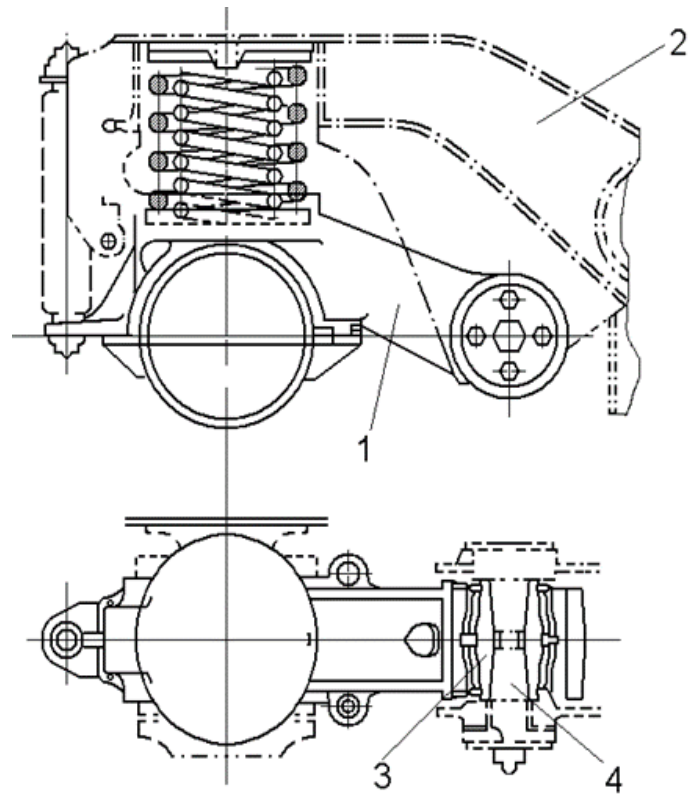

Fig. 1. System with articulated drive arm with a silent block with possibilities for adjusting the elasticity.

The mechanical model of an elastic driving bogie used in the study is shown in fig. 2 .

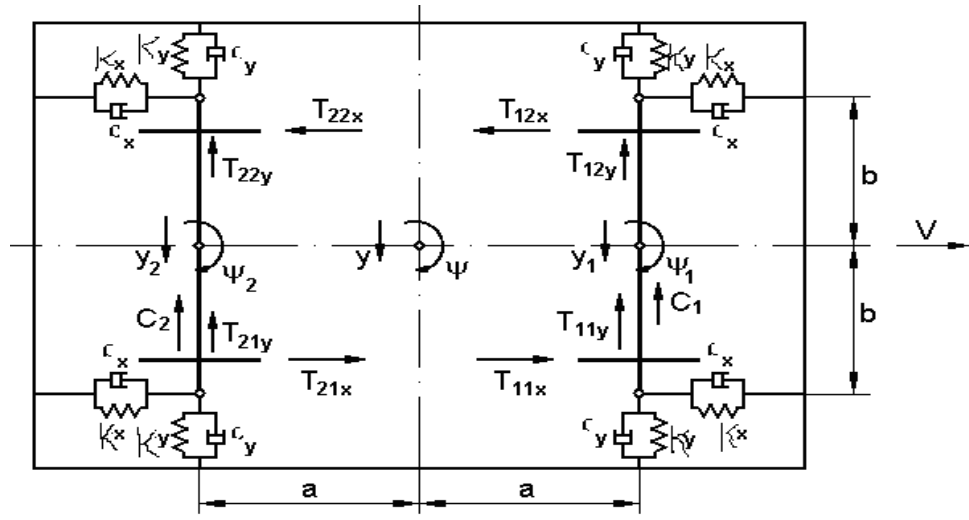

Fig. 2. Mechanical model of the bogie with the forces acting on the elastically driven axles.

For an elastic driving bogie axle, we will analyse the inclusion curve assuming a quasistatic stationary motion regime. A reference work that deals with this problem has been developed by D. E. Newland [1]. It is supposed that under the action of external Fn and contact forces between wheels also rails, bogie is positioned in curve in Fig. 3, axles makes with normals to curve (attack) $\alpha_{1}$ and that respectively $\alpha_{2}$. Also, it supposed that there are 
no large sliding of wheels (with profile wear) but pseudo sliding proportional with to contact forces and that forces balance can ensure running of axles without contact on lips (of wheelsets).

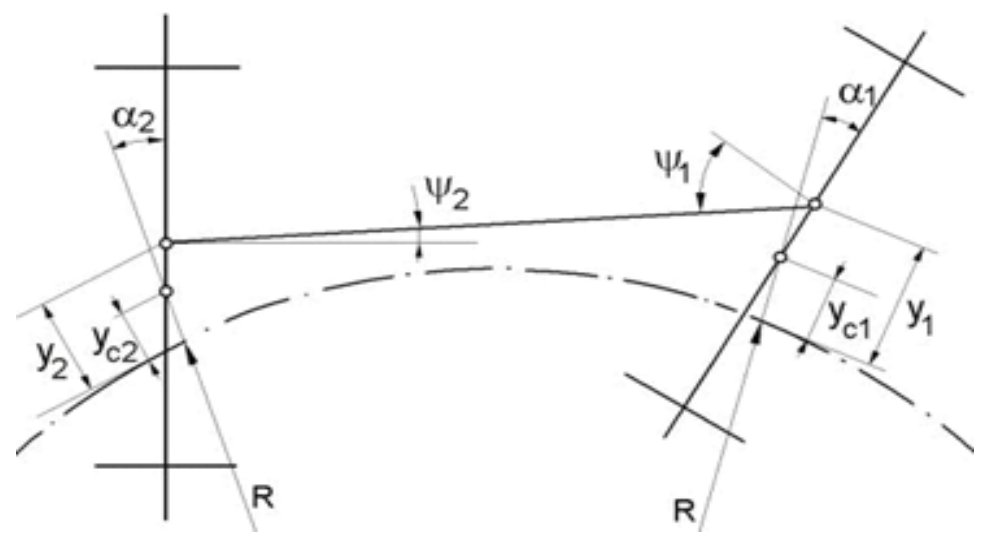

Fig. 3. Driving axle bogie spring curved movements.

To the axis of the track, the axle centers are lagged outside with $y_{c 1}$ and, respectively, $y_{c 2}$ and the bogie chassis reduced to its longitudinal axis, is lagged with the right axle $y_{l}$ and, respectively, $y_{2}$. During the movement, the forces of friction, forces of centering and the forces from guidance system, of each axle, must be balanced.

In front of the chassis axles are rotated with $\psi_{12}$.

On the axes act on the friction forces $T_{x}, T_{y}$, the centering forces $C$, forces in the guidance system dependent on the elastic constants $\mathrm{k}_{\mathrm{x}}, \mathrm{k}_{\mathrm{y}}$ as well as an outside force transmitted via bogie system - vehicle bodywork.

The positions of the axles in the curves result from the equilibrium conditions of the forces acting on the two axles and the bogie frame respectively. Friction coefficients between wheels and rails were evaluated on the basis of Kalker's theory [4]. Details of equilibrium equations are presented in detail in the paper [1]

The theoretical study shows that an axle-driven bogie will run without slipping on any curve whose radius is:

$$
R \geq \frac{\chi}{2 C \mu} \sqrt{1+\frac{a^{2}}{4 \cdot e^{2}} A^{2}}
$$

where $\chi$-is the pseudo-slip coefficient $\left(\chi_{x}=\chi_{y}=\chi\right) ; \mu$-coefficient of adhesion; $\mathrm{e}-$ semi-track gauge; $\gamma$ - being the actual conicity of the wheel profile and $\mathrm{Q}$ - wheel load.

$$
A=1+\frac{\chi Q}{2 c_{x} b^{2}} \cdot \frac{e \gamma}{r}\left(1+\frac{4 c_{x} b^{2}}{c_{y} a^{2}}\right)
$$




$$
C=1+\frac{(\chi Q)^{2} a}{\left(2 c_{x} b^{2}\right)^{2}} \cdot \frac{e \gamma}{r}\left(1+\frac{4 c b^{2}}{c_{y} a^{2}}\right)
$$

The more elastic the suspension, the smaller the radius $\mathrm{R}$ of the curve, the axes tilting to a radial position.

Comparing the displacements of lateral force $F_{n}$, is observed that the rear axle is shifted more than the front axle, this movement being independent of the radius of the curve.

The displacement under the effect of force $F_{n}$ is also independent of the deviation from the track center line, which occurs even if the bogie side not exercise any power and actually indicate the inherent ability to be self-guided bogie in way pseudo sliding forces between wheels and rails.

\section{The influence elasticities from conduction system of the axles on the bogie hunting stability}

The study stability motion of winding bogie axle with elastic driving axis is based on relationships obtained after linearization phenomenon of winding. Linearization winding phenomenon is realized taking in consideration that the contact forces vary linearly with lateral movement of the axle also, neglecting friction and games of various elements of the bearing structure of the vehicle and neglecting tread irregularities and discontinuities. Not least taking in consideration equivalent conicity wheel profile as constant and proportional to the tangential force pseudo sliding point of contact of the wheel with the rail. [3], [5]

This study aims to determine the velocity at which the stable movement of winding vehicle equipped with elastic driving axle bogies will turn into an unstable movement, to determine the establishment of critical speed, which when exceeded will result in a rapid deterioration walking. In other words, we aimed to determine the maximum speed that can be reached safely by vehicle. Consider the case of general movement of the bogie in winding which the suspension consists in arches having elastic constants $k_{x}, k_{y}$, and the linear characteristic of the shock absorbers (non-viscous), which amortizations constants $\mathrm{c}_{\mathrm{x}}$ and $\mathrm{c}_{\mathrm{y}}$ (Fig.2).

Center of mass of the bogie is considered located in the axle axles. Determination of critical speed and critical pulse respectively when is neglect the suspended mass of bogie and of amortizations, can be based on the equations of movement for the bogie frame and axles, neglecting balance of axle, the effect of spin and gyroscopic noted: $k_{x}$ and $k_{y}$ - the elastic constants in the longitudinal and transverse, $m_{o}$ - the wheelset (axle) mass, $Q$ - the wheel load, $a$ - the wheelbase bogie, $b$ - the transverse cross midway between the suspension arches $e$ the midway between the nominal rolling circles, $r$ - the wheel ray (radius), $v$ - the velocity running speed, $\gamma$ - equivalent conicity.

The model studied was applied to the bogies of towed vehicles by neglecting the suspended bogies and shock absorbers.

For the rail wheel friction coefficient, it can be considered the work of $\mathrm{P}$ van Bommel [3] which recommends some approximate values of the pseudo-slip coefficient based on the relations established by Kalker.

In the general motion case, the stability study and the determination of the amplitudes of the repositioning oscillations can be done by applying a numerical method of integrating the motion equations by means of the calculation technique. With the above assumptions, the oscillating system can be reduced to 4 degrees of freedom. In this case, the critical velocity $v_{c}$, 
respectively the critical pulse $\omega_{\mathrm{c}}$, is determined with simple relations derived from the equation of the pulsations of the system [1].

Thus, the characteristic equation of the system is:

$$
\left|\begin{array}{cccc}
T_{1}(p) & 0 & -4 \chi Q & 0 \\
0 & T_{1}(p)+4 c^{*} & -4 c^{*}{ }^{*} a & -4 \chi Q \\
-4 \chi Q \frac{e \gamma}{r} & -4 c^{*}{ }^{a} & T_{2}(p)+4 c^{*} a^{2} & 0 \\
0 & 4 \chi Q \frac{e \gamma}{r} & 0 & T_{2}(p)+4 c^{*} b^{2}
\end{array}\right|=0
$$

where $c_{y}^{*}=\frac{c_{x} c_{y} b^{2}}{c_{y} a^{2}+c_{x} b^{2}} ; T_{1}(p)=2 m_{o} p^{2}+\frac{4 \chi Q}{v} \mathrm{p} ; T_{2}(p)=2 m_{o} e^{2} p^{2}+\frac{4 \chi Q e^{2}}{v} \mathrm{p}$

Considering that the stability limit has been reached when $v=v_{c}$, substituting $p=j \omega_{c}(j=-1)$ results the relations of critical speed determination.

\section{Numeric application. Experimental determinations}

With the constructional characteristics of the Y32 bogie, which equips the passenger coaches in the CFR park, it results that for the elastic constants of the axle $k_{x}=10^{7} \mathrm{~N} / \mathrm{m}$ and $k_{y}=5 \cdot 10^{7} \mathrm{~N} / \mathrm{m}$ it results that it runs without slipping of the wheels on the track if the radius of the curve is $\mathrm{R} \geq 1552 \mathrm{~m}$.

Studying the stability of the vehicle on straight-line traffic resulted in safe traffic up to critical speed $\mathrm{v}_{\mathrm{c}}=64 \mathrm{~m} / \mathrm{s}=230 \mathrm{~km} / \mathrm{h}$.

A series of experimental determinations were made at the Romanian Railway Authority (AFER) in Bucharest and Softronic Craiova. On the Făurei ring, the bogie Y32 was experimentally resulting in a stable movement up to $210 \mathrm{~km} / \mathrm{h}$.

Experimental determinations have been made at Softronic on special stands for rail vehicle suspensions.

In fig. 4 there is shown the stand for determining the elastic characteristics and dynamic operation of the suspension elastic element.

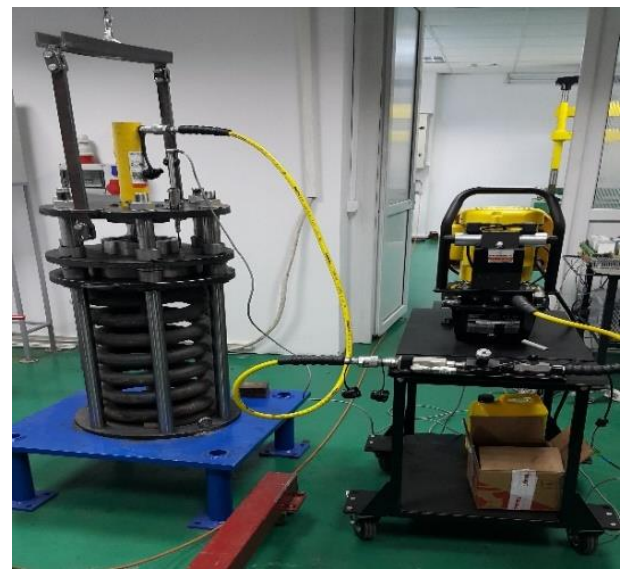

Fig. 4. Stand for determining elastic features. 


\section{Conclusions}

From the curve flow study is obtained a minimum radius value for not occurring sliding on the wheel - rail contact. In this case, there is a tendency of radial positioning of the first axle. The relationship shown shows the advantages of radial guidance in the case of curves with low radius, but with an appropriate adaptation of the axle guidance system of the vehicle.

Also results of the study have led to the conclusion that, when the speed of about $230 \mathrm{~km} / \mathrm{h}$ is exceeded, the vehicle's winding movement will become unstable, that leading to inadmissible transverse stresses of the runway and even endangering the safety circulation. The maximum safe running speed of a vehicle shall be less than $10-15 \%$ from critical shock velocity if a possible change in time of the elastic characteristics of the axle guidance system is considered.

The presented relations allow to analyze the influence of the various constructive parameters of the bogie on the winding movement and to determine the constructive conditions for expansion at higher winding stability. Although the applied method is based on a series of simplifying hypothesis, it can be used for rapid engineering evaluations of bogie performances.

The calculation model belongs to the author of the paper and it is experimentally validated on a series of high-speed bogies analyzed in the Railway Rolling Stock Department from the Polytechnic University of Bucharest.

Experiments have also been carried out at AFER as well as at Softronic Craiova. They validated the theoretical semi-distances.

This work was supported by a grant of the Romanian National Authority for Scientific Research and Innovation, CNCS-UEFISCDI, project number PN-III-P2-2.1-PTE-2016-0008, within PNCDI III

\section{References}

1. D.E. Newland, Steering a Flexible Railway Track in Curved Track. (Transaction of ASME 1969)

2. $\mathrm{P}$. van Bommel, Considerations lineaires concernant le mouvement de lacet d'un vehicule ferroviaire. (UIC/ORE,C9 1968)

3. J.J. Kalker, Three-dimensional elastic bodies in rolling contact.Kluwer (Academic Publishers, Dortrech E.A. 1990)

4. R. Joly, Rail International, 12 (1971)

5. R Joly, M. Laurent, Etude de la dynamique transversal d'un vehicule ferroviaire. Banc experimental de Vitry, (Rapport SNCF, division des essays de materiel 1974) 